\title{
openheart Superiority of proatrial natriuretic peptide in the prognostic power in patients with acute decompensated heart failure on hospital admission: comparison with B-type natriuretic peptide and other natriuretic peptide forms
}

Seiji Takashio, ${ }^{1}$ Hiroyuki Takahama, ${ }^{1}$ Toshio Nishikimi, ${ }^{2}$ Tomohiro Hayashi, ${ }^{1}$ Chiaki Nagai-Okatani, ${ }^{3}$ Ayaka Matsuo, ${ }^{4}$ Yasuaki Nakagawa, ${ }^{2}$ Masashi Amano, ${ }^{1}$ Yasuhiro Hamatani, ${ }^{1}$ Atsushi Okada, ${ }^{1}$ Makoto Amaki, ${ }^{1}$ Takuya Hasegawa, ${ }^{1}$ Hideaki Kanzaki, ${ }^{1}$ Satoshi Yasuda, ${ }^{1}$ Kenji Kangawa,${ }^{5}$ Toshihisa Anzai, ${ }^{1,6}$ Naoto Minamino, ${ }^{3,4}$ Chisato Izumi ${ }^{1}$

- Additional material is published online only. To view please visit the journal online (http://dx.doi.org/10.1136/ openhrt-2019-001072).

To cite: Takashio $\mathrm{S}$, Takahama H, Nishikimi T, et al. Superiority of proatrial natriuretic peptide in the prognostic power in patients with acute decompensated heart failure on hospital admission: comparison with B-type natriuretic peptide and other natriuretic peptide forms. Open Heart 2019;6:e001072. doi:10.1136/ openhrt-2019-001072

Received 20 April 2019 Revised 5 June 2019 Accepted 13 June 2019

Check for updates

(c) Author(s) (or their employer(s)) 2019. Re-use permitted under CC BY-NC. No commercial re-use. See rights and permissions. Published by BMJ.

For numbered affiliations see end of article.

\section{Correspondence to} Dr Hiroyuki Takahama, National Cerebral and Cardiovascular Center, Suita 564-8565, Japan; takahama@ncvc.go.jp

\section{ABSTRACT}

Aims There are significant differences in how atrial (A-type) and B-type natriuretic peptide (ANP and BNP) are secreted and metabolised, but there is little information available about the relative clinical significance of the two peptides. The aim of the present study was to investigate: (1) the association between the circulating level of each ANP molecular form and patient clinical background and (2) their prognostic power for patients with acute decompensated heart failure (ADHF).

Methods We used specific chemiluminescence enzyme immunoassays to prospectively evaluate the levels of six bioactive molecular forms of ANP (pro-ANP, $\beta$-ANP and total ANP) and BNP (pro-BNP, N-terminal pro-BNP (NT-proBNP) and total BNP) in plasma samples collected from 173 patients with ADHF on their hospital admission.

Results We found that pro-ANP levels were strongly associated with left ventricular (LV) size and ejection fraction $(p<0.001)$, but were not associated with left atrial size. Percent pro-ANP ([pro-ANP/total ANP]x100) was also associated with LV size and function. During the follow-up term (median: 469 days), composite adverse events (all causes of death or rehospitalisation for HF) occurred in 67 patients (38.7\%). Pro-ANP was significantly associated with composite adverse events even after adjusting by estimated glomerular filtration rate (eGFR) $(p<0.05)$. In contrast, NT-pro-BNP was not independent of eGFR in the multivariate analysis.

Conclusion Circulating levels of pro-ANP are strongly associated with LV function and clinical outcomes of patients with ADHF. These findings suggest that during the acute phases of HF, pro-ANP has a prognostic power comparable with NT-pro-BNP independently of renal function.

\section{Key messages}

What is already known about this subject?

- B-type natriuretic peptide (BNP) or N-terminal proBNP (NT-pro-BNP) levels during chronic phases of heart failure (HF), such as those levels at discharge from hospital, are now well established as prognostic biomarkers of HF. But BNP on admission is less predictive in patients with acute decompensated $\mathrm{HF}$ (ADHF). Thus, on admission: in a vulnerable state of ADHF, there is still an unmet need for a biomarker for prediction of future clinical outcomes. Little has been known about the prognostic power of each natriuretic peptide molecular form including atrial natriuretic peptide (ANP) in patients with ADHF. We therefore, compared predictability of circulating all measurable molecular forms of ANP and BNP for clinical outcome in patients with ADHF.

What does this study add?

- In this study, we were able to measure circulating levels of six molecular forms of ANP and BNP, including pro-ANP, $\beta$-ANP, total ANP, total BNP, NT-pro-BNP and pro-BNP during the acute phase of HF. Our findings showed that only pro-ANP on admission among the six measured natriuretic peptide forms was significantly associated with composite adverse events independently of renal function. We also found that pro-ANP levels were strongly associated with left ventricular size and ejection fraction.

\section{INTRODUCTION}

The natriuretic peptide system consists of three of peptide factors: atrial (A-type) 


\section{Key messages}

How might this impact on clinical practice?

- These findings suggest that during the acute phase of HF, pro-ANP is a more predictive biomarker with independency of renal function compared with NT-pro-BNP, will be one of candidates of biomarker in acute phases of HF.

natriuretic peptide (ANP) and the B-type and C-type natriuretic peptides (BNP and CNP). Whereas CNP is produced in the bone, central nervous system and vascular endothelium, BNP is predominantly produced from the ventricular myocardium, and ANP is mainly from the atrial myocardium. ${ }^{1}$ Pro-ANP, the precursor of mature and fully bioactive $\alpha$-ANP, is stored in secretory granules in atrial cardiomyocytes and it is cleaved into bioactive $\alpha$-ANP and amino N-terminal pro-ANP (NT-pro-ANP) by a transmembrane proteolytic enzyme, corin, when it is secreted. Earlier studies also revealed that an antiparallel dimer of $\alpha$-ANP ( $\beta$-ANP) is detectable in the plasma of patients with heart failure (HF), along with $\alpha$-ANP and pro-ANP. ${ }^{23}$ In contrast to ANP, pro-BNP is cleaved intracellularly into mature, fully bioactive BNP-32 and an (N-terminal pro-BNP) NT-pro-BNP by the enzyme furin. We and others previously reported that the molar ratio of pro-BNP to total BNP (sum of mature BNP plus pro-BNP) is decreased in patients with acute $\mathrm{HF}^{4}$ or milder acute $\mathrm{HF}^{5}$ and that it is increased in patients with severe chronic $\mathrm{HF}(\mathrm{CHF})^{6}$ elevated in patients with severe acute HF.

In contrast to BNP, little is known about the circulating levels of ANP molecular forms or the regulation of their processing. Moreover, the clinical significance of their plasma levels and the molar ratio of pro-ANP to total ANP (sum of mature ANP, $\beta$-ANP and pro-ANP) in patients with HF remain unclear. In fact, there has been controversy regarding the distribution of ANP molecular forms in the plasma of patients with HF. Using radioimmunoassays, Miyata $e t a l^{7}$ found that $\alpha$-ANP is the major circulating form in patients with HF. On the other hand, Wei et $a l^{2}$ reported that $\beta$-ANP is the major form, accounting for $61 \%$ of the total plasma ANP in patients with HF. Although the precise reason for the discrepancy between the two studies is not known, it likely derives from differences in the methods employed (with or without extraction of plasma, different antibodies used for radioimmunoassays, different columns or separation conditions for chromatography). Furthermore, the clinical significance of the ANP molecular profile in the plasma has been only minimally investigated. To address these issues, we recently developed chemiluminescent enzyme immunoassays (CLEIAs) specific for total ANP, pro-ANP and $\beta$-ANP. ${ }^{8}$

BNP is an established biomarker predictive of future clinical events in patients with HF. However, plasma BNP levels measured on admission, when the patient is in a vulnerable state of acute decompensated HF (ADHF), are less predictive than at discharge, when the patient is clinically stable. $^{9-11}$ Given that optimal disease management and decision-making about treatment strategy require accurate clinical risk stratification on admission, the prognostic power of known biomarkers is not sufficient in patients with ADHF. We, therefore, compared circulating levels of all measurable molecular forms of ANP and BNP in patients with ADHF. Our aims were to (1) investigate the associations of levels of total ANP and each ANP molecular form with clinical background; (2) assess the prognostic power of pro-ANP, $\beta$-ANP, total ANP, pro-BNP, NT-pro-BNP and total BNP measured in patients with ADHF on hospital admission and (3) investigate the ratio of pro-ANP to total ANP as an index of unprocessed pro-ANP rate.

\section{METHODS \\ Study design}

This was a prospective cross-sectional study to measure the plasma levels of all measurable molecular forms of ANP and BNP in patients with ADHF admitted to our hospital.

\section{Study population}

Eligibility requirements were as follows: (1) age from 20 to 85 years, (2) admission to our hospital for treatment of ADHF, (3) enrolment within 48 hours after admission for ADHF and (4) provided written informed consent. Patients on dialysis were excluded, as were patients with acute coronary syndrome. In this study, patients who received carperitide (recombinant $\alpha$-human ANP) before blood collection on admission were excluded. Ultimately, we analysed 173 patients (aged 26-85 years) enrolled between June 2012 and October 2015.

\section{Biomarker testing}

Details of the measurement procedures are described in the online supplementary methods.

\section{Measurement of two BNP forms}

Plasma total BNP and pro-BNP were measured using CLEIAs as previously described. ${ }^{512}$ NT-pro-BNP concentrations were measured using the Elecsys pro-BNP II assay (Roche Diagnostics, Basel, Switzerland). Details of the measurement procedures are described in the online supplementary methods.

\section{Measurement of three ANP forms}

Plasma total ANP, pro-ANP and $\beta$-ANP were measured using our recently developed plate-based CLEIAs. ${ }^{8}$ In brief, total ANP was measured with a sandwich CLEIA using antibodies recognising the C-terminal tail and the ring portion of ANP as the capture and detection antibodies, respectively. The limit of quantification (LOQ) was calculated to be $0.26 \mathrm{pM}$. Intra-assay (20 replicates) and interassay (20 replicates) coefficients of variation (CV) of the standard at seven different concentrations were in the range of $0.9 \%-4.6 \%$ and $2.5 \%-9.0 \%$, 
respectively. Pro-ANP was quantitated using the same method with antibodies recognising the ring portion and N-terminal region of the peptide as capture and detection antibodies, respectively. The LOQ was 0.21 pM. Intra-assay and interassay CVs determined under the same conditions used for total ANP were in the range of $1.2 \%-3.7 \%$ and $3.0 \%-8.6 \%$, respectively. For these two assays, plasma samples were diluted by twofold or more and directly measured using the CLEIAs. Recombinant FLAG-pro-ANP was used as the standard. It was custommade by ProCube (Kobe, Japan), purified through size-exclusion and reverse phase-high-performance liquid chromatography, and quantified by amino acid analysis.

The CLEIA for $\beta$-ANP was performed using the same method with an antibody recognising the ring portion as the capture antibody, and another specifically recognising the three-dimensional structure of the molecule as the detection antibody. Its LOQ was $0.23 \mathrm{pM}$. Intra-assay and interassay CVs determined under the same conditions used for total ANP were in the ranges of $0.3 \%-$ $2.8 \%$ and $4.0 \%-6.2 \%$, respectively. In this assay, plasma samples were desalted and extracted using Sep-Pak C18 Plus cartridges (Waters, Milford, USA), as reported previously. ${ }^{8}$ The eluate was lyophilised, reconstituted in the sampling buffer and applied to the $\beta$-ANP CLEIA. Quantified synthetic $\beta$-ANP (Peptide Institute, Osaka, Japan) served as the standard.

In the total ANP CLEIA, $\alpha$-ANP, $\beta$-ANP and FLAGpro-ANP were measured at a ratio of 1:2:1 based on the number of $\alpha$-ANP units. In the pro-ANP CLEIA, $\alpha$-ANP and $\beta$-ANP showed less than $0.4 \%$ cross-reactivity, while $\alpha$-ANP and FLAG-pro-ANP showed less than $1.1 \%$ cross-reactivity in the $\beta$-ANP CLEIA. These three CLEIAs had no cross-reactivity with BNP-32 or CNP-22. ${ }^{8}$

Total ANP is sum of pro-ANP, $\alpha$-ANP and beta ANP. Per cent pro-ANP is calculated from the following equation: $\%$ pro-ANP $=[$ pro-ANP $/$ total ANP]X100.

\section{Cyclic guanosine monophosphate and other laboratory markers}

Details of the measurement procedures are described in the online supplementary methods.

\section{Echocardiography}

Echocardiography was routinely performed when the patients were admitted to the hospital. Details of the measurement procedures are described in the online supplementary methods.

\section{Diagnosis of ADHF}

ADHF was diagnosed using the Framingham criteria. ${ }^{13}$ The final diagnosis of cardiomyopathy was based on the definition from WHO/International Society and Federation of Cardiology Task Force. ${ }^{14}$

\section{Clinical outcomes}

After the admission date, we investigated all causes of death, implantation of a left ventricular (LV) assist device, and rehospitalisation for $\mathrm{HF}$ for 2 years through medical chart review or a letter. Combined clinical events were defined as all-cause death, implantation of an LV assist device or rehospitalisation for HF.

\section{Statistical analysis}

Data are expressed as medians and IQR. Fisher's exact test or the $\chi^{2}$ statistic was used to compare categorical variables, as appropriate. For baseline patient characteristics, Wilcoxon's rank-sum test was used for comparison of continuous variables between two groups. Univariate and multivariate survival analyses were performed using the Cox proportional hazards model. HRs with 95\% CI and probability $(p)$ values determined using the likelihood ratio test are presented. A multiple linear regression model was used to test multiple covariates. All tests were two tailed, and $\mathrm{p}<0.05$ was considered significant. All statistical analyses were performed using JMP V.9 statistical analysis software (SAS Institute Japan).

\section{RESULTS}

\section{Patient characteristics}

The enrolled patients with ADHF on admission were characterised as follows: 95\% were New York Heart Association class III-IV on admission; the median LV ejection fraction (LVEF) was 34\% (IQR: 23, 50\%), median LV end-diastolic diameter (LVDd) was $56 \mathrm{~mm}$ (IQR: 47-64 $\mathrm{mm})$ and median hospitalisation length was 22 days (IQR: 14-36 days).

The histograms in figure 1 show the patient distribution plotted against the measured concentrations of each ANP form. In addition, online supplementary table 1 summarises the baseline characteristics of patients whose plasma levels of each ANP molecular form were below or above the median. Patients with above median of these three ANP molecular forms (total ANP, $\beta$-ANP and pro-ANP) commonly presented with lower systolic blood pressure, larger LV size, lower LVEF and higher total BNP levels than those with below median levels. Similar tendencies (larger LV size and lower LVEF) were seen in patients with higher levels of total BNP and pro-BNP $(\mathrm{p}<0.05)$.

\section{Prognostic power of plasma natriuretic peptide levels on admission in patients with ADHF}

During the follow-up term (median: 469 days), composite clinical adverse events (all causes of death $(n=27)$ or rehospitalisation for HF $(\mathrm{n}=60))$ occurred in 67 patients $(38.7 \%)$. Table 1 shows the association between the plasma level of each natriuretic peptide form and clinical outcomes. Pro-ANP and NT-pro-BNP levels were associated with clinical outcomes in patients with ADHF. With the exception of pro-ANP and NT-pro-BNP, no natriuretic peptide form was significantly associated with the composite clinical adverse events. Moreover, multivariate analysis showed that pro-ANP levels were associated with composite clinical outcomes, even after adjusting for age, sex (table 1B), body mass index (table 1C) and estimated glomerular filtration rate (eGFR) (table 1D). In contrast, 

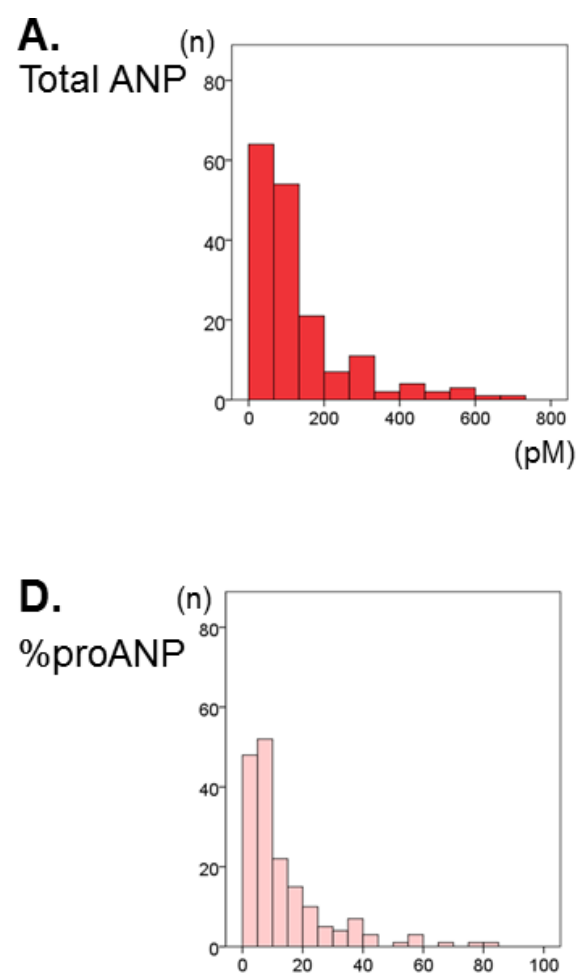
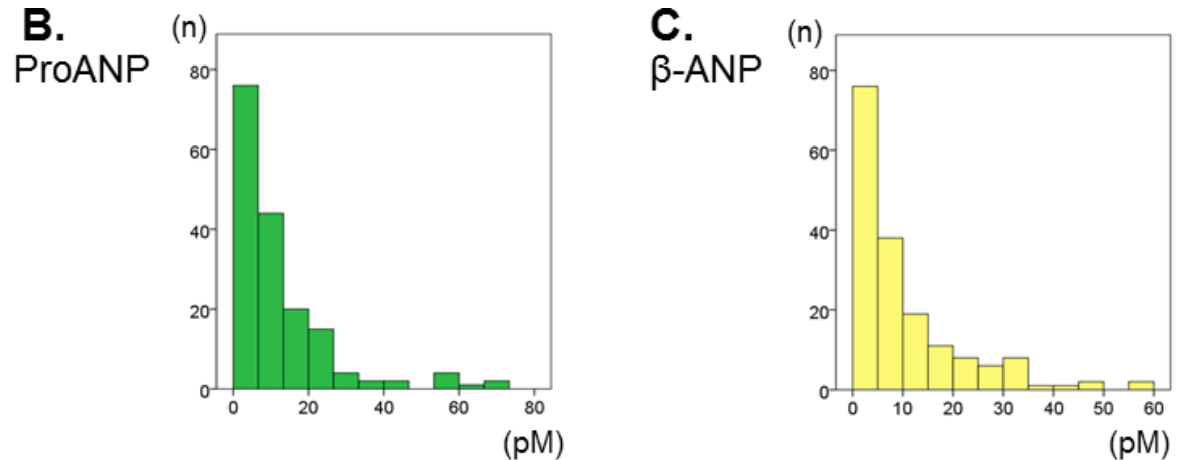

E.

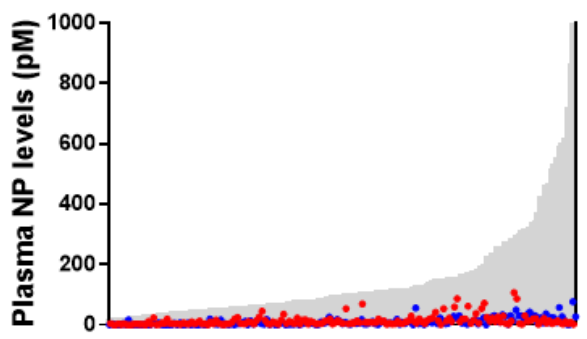

Table 1 Predictive values of natriuretic peptides (NPs) on admission for future clinical adverse events

\begin{tabular}{|c|c|c|c|}
\hline Variables & HR & $95 \% \mathrm{Cl}$ & P value \\
\hline \multicolumn{4}{|l|}{ (A) Univariate analysis } \\
\hline Total ANP (per 10 pM) & 1.01 & 1.00 to 1.02 & 0.108 \\
\hline$\beta$-ANP (per 10 pM) & 1.14 & 0.93 to 1.37 & 0.185 \\
\hline Pro-ANP (per 10 pM) & 1.18 & 1.03 to 1.34 & 0.018 \\
\hline BNP (per 10 pM) & 1.02 & 0.99 to 1.03 & 0.101 \\
\hline Pro-BNP (per 10 pM) & 1.03 & 1.00 to 1.05 & 0.058 \\
\hline NT-pro-BNP (per 10 pM) & 1.00 & 1.00 to 1.01 & 0.002 \\
\hline \multicolumn{4}{|c|}{ (B) Age and sex-adjusted model } \\
\hline Pro-ANP (per 10 pM) & 1.19 & 1.03 to 1.34 & 0.018 \\
\hline NT-pro-BNP (per 10 pM) & 1.00 & 1.00 to 1.01 & 0.002 \\
\hline \multicolumn{4}{|c|}{ (C) Age, sex and BMI-adjusted model } \\
\hline Pro-ANP (per 10 pM) & 1.07 & 1.02 to 1.11 & 0.001 \\
\hline NT-pro-BNP (per 10 pM) & 1.00 & 1.00 to 1.01 & 0.001 \\
\hline \multicolumn{4}{|c|}{ (D) Age, sex and eGFR-adjusted model } \\
\hline Pro-ANP (per 10 pM) & 1.05 & 1.00 to 1.10 & 0.046 \\
\hline NT-pro-BNP (per 10 pM) & 1.00 & 1.00 to 1.01 & 0.062 \\
\hline
\end{tabular}

Values expressed per $10 \mathrm{pM}$ increases of each NP.

ANP, A-type natriuretic peptide;BMI, body mass index; BNP,

B-type natriuretic peptide; eGFR, estimated glomerular filtration rate. multivariate analysis adjusted by eGFR showed that NT-pro-BNP was not associated with clinical outcomes (table 1D).

\section{Association between \%pro-ANP and clinical background}

Because of the strong association between plasma levels of pro-ANP and clinical outcome, we next assessed \%proANP. Table 2 shows the clinical characteristics of patients whose plasma \% pro-ANP levels were below or above the median (median \%pro-ANP $=9.0 \%$ ). A history of $\mathrm{HF}$ hospitalisation was frequently observed in patients with above-median \% pro-ANP levels. In addition, patients with above-median \% pro-ANP levels were characterised with larger LV size, lower LVEF, lower eGFR, lower body mass index, high-sensitive cardiac troponin $\mathrm{T}$ levels and higher plasma BNP levels. Medications such as beta-blockers and aldosterone antagonists were more frequently taken by patients with above-median \%pro-ANP. In addition, the frequency of persistent atrial fibrillation was tended to be higher in the above-median group than below-median $\%$ pro-ANP group, but was statistically no significant (52\% vs $46 \%$, respectively).

We next examined the associations between cardiac function/structure and the plasma concentration of each ANP molecular form (table 3). Plasma total ANP were not associated with LVDd, LVEF or left atrial diameter (LAd). By contrast, pro-ANP levels associated significantly with LVEF $(p<0.0001)$ and LVDd $(p<0.001)$, but not with LAd. 
Table 2 Baseline characteristics of patients with below/above median \%pro-ANP

\begin{tabular}{|c|c|c|}
\hline & Below median \%pro-ANP (<9.0\%) & $\begin{array}{l}\text { Above median \%pro-ANP } \\
(\geq 9.0 \%)\end{array}$ \\
\hline Patient number & 86 & 87 \\
\hline Age (years) & $75(66,80)$ & $76(68,80)$ \\
\hline Gender (female \%) & $28(33)$ & $32(37)$ \\
\hline $\mathrm{BMI}\left(\mathrm{kg} / \mathrm{m}^{2}\right)$ & $24.3(21.6,26.6)$ & $22.2(20.0,24.8)^{\star}$ \\
\hline \multicolumn{3}{|l|}{ NYHA class } \\
\hline Class III or IV, N (\%) & $84(98)$ & $80(92)$ \\
\hline \multicolumn{3}{|l|}{ Aetiology } \\
\hline Ischaemic, N (\%) & $21(24)$ & $32(37)$ \\
\hline Non-ischaemic, N (\%) & $14(16)$ & $24(28)$ \\
\hline Valvular, N (\%) & $25(29)$ & $13(15)$ \\
\hline Hypertensive, N (\%) & $18(21)$ & $14(16)$ \\
\hline Others, N (\%) & $8(9)$ & $4(5)$ \\
\hline \multicolumn{3}{|l|}{ History } \\
\hline HF hospitalisation, N (\%) & $31(36)$ & $46(53)^{*}$ \\
\hline Hypertension, N (\%) & $59(68)$ & $58(67)$ \\
\hline DM, N (\%) & $36(41)$ & $35(41)$ \\
\hline Dyslipidaemia, N (\%) & $46(53)$ & $39(45)$ \\
\hline \multicolumn{3}{|l|}{ Vital signs on admission } \\
\hline Systolic blood pressure (mm Hg) & $138(118,153)$ & $130(108,159)$ \\
\hline Heart rate (bpm) & $91(73,111)$ & $80(70,97)^{\star}$ \\
\hline \multicolumn{3}{|l|}{ Echocardiography } \\
\hline $\operatorname{LVDd}(\mathrm{mm})$ & $54(47,60)$ & $58(48,67)^{\star}$ \\
\hline LVDs (mm) & $40(32,52)$ & $48(37,60)^{*}$ \\
\hline LVEF (\%) & $40(25,53)$ & $31(20,47)^{\star}$ \\
\hline LADs (mm) & $46(40,51)$ & $50(42,54)$ \\
\hline \multicolumn{3}{|l|}{ Laboratory data } \\
\hline Haemoglobin $(\mathrm{g} / \mathrm{dL})$ & $12.5(10.4,13.5)$ & $11.9(10.6,13.4)$ \\
\hline eGFR (mL/min/1.73 m²) & $50.4(37.2,64.9)$ & $39.7(29.7,54.5)^{\star}$ \\
\hline BUN (mg/dL) & $21(17,28)$ & $26(20,34)^{\star}$ \\
\hline $\mathrm{CRP}(\mathrm{mg} / \mathrm{dL})$ & $0.42(0.17,1.21)$ & $0.41(0.10,1.94)$ \\
\hline hs-cTnT (ng/mL) & $0.030(0.017,0.053)$ & $0.040(0.026,0.072)^{*}$ \\
\hline Plasma aldosterone concentration (ng/dL) & $74.9(37.1,131.0)$ & $86.6(48.9,186.1)$ \\
\hline Total plasma BNP (pmol) & $93.9(44.1,162.7)$ & $138.8(81.1,201.5)^{\star}$ \\
\hline Cyclic GMP (pmol/mL) & $15.0(6.4,20.2)$ & $12.0(8.4,16.0)$ \\
\hline \multicolumn{3}{|l|}{ Medications } \\
\hline ACEi or ARB, N (\%) & $55(64)$ & $52(60)$ \\
\hline Beta-blockers, N (\%) & $55(64)$ & $62(71)$ \\
\hline Aldosterone antagonists, N (\%) & $27(31)$ & $37(43)$ \\
\hline Loop diuretics, N (\%) & $52(60)$ & $59(68)$ \\
\hline
\end{tabular}

Values are the median (IQR) and patients number, $\mathrm{N}(\%)$.

${ }^{*} \mathrm{P}<0.05$ vs below median \%pro-ANP.

$\mathrm{ACEi}$, angiotensin-converting enzyme inhibitor; ANP, atrial (A-type) natriuretic peptide; ARB, angiotensin II receptor blocker; BMI, body mass index; BNP, B-type natriuretic peptide; BUN, blood urea nitrogen; CRP, C reactive protein; DM, diabetes mellitus; GMP, guanosine monophosphate; HF, heart failure; hs-TnT, high sensitive cardiac troponin T; LAd, left atrial diameter; LVDd, left ventricular end-diastolic diameter; LVDs, left ventricular end-systolic diameter; LVEF, left ventricular ejection fraction; NYHA, New York Heart Association; eGFR, estimated glomerular filtration rate. 
Table 3 Relationships of natriuretic peptide molecular forms with cardiac structure and function

\begin{tabular}{|c|c|c|}
\hline & $r$ & $P$ value \\
\hline \multicolumn{3}{|l|}{ LVEF } \\
\hline Total ANP & 0.155 & 0.441 \\
\hline$\beta$-ANP & 0.192 & 0.017 \\
\hline Pro-ANP & 0.302 & $<0.0001$ \\
\hline Total BNP & 0.423 & $<0.0001$ \\
\hline Pro-BNP & 0.404 & $<0.0001$ \\
\hline NT-pro-BNP & 0.244 & 0.001 \\
\hline \multicolumn{3}{|l|}{ LVDd } \\
\hline Total ANP & 0.058 & 0.473 \\
\hline$\beta$-ANP & 0.091 & 0.255 \\
\hline Pro-ANP & 0.262 & $<0.001$ \\
\hline Total BNP & 0.269 & 0.0004 \\
\hline Pro-ANP & 0.279 & 0.0002 \\
\hline NT-pro-BNP & 0.084 & 0.273 \\
\hline \multicolumn{3}{|l|}{ LAd } \\
\hline Total ANP & 0.075 & 0.461 \\
\hline$\beta$-ANP & 0.111 & 0.272 \\
\hline Pro-ANP & 0.175 & 0.083 \\
\hline Total BNP & 0.150 & 0.138 \\
\hline Pro-BNP & 0.156 & 0.123 \\
\hline NT-pro-BNP & 0.108 & 0.289 \\
\hline
\end{tabular}

ANP, atrial (A-type) natriuretic peptide; BNP, B-type natriuretic peptide; LAd, left atrial diameter; LVDs, left ventricular end-systolic diameter; LVEF, left ventricular ejection fraction; NT-pro-BNP, $\mathrm{N}$-terminal fragment of pro-BNP.

Plasma levels of $\beta$-ANP were associated with LVEF. Additionally, both total BNP and pro-BNP levels associated with LV size and function $(\mathrm{p}<0.0001)$ (table 3$)$.

\section{Clinical determinants for \%pro-ANP in patients with ADHF}

Table 4 shows the univariate/multivariate analysis of $\%$ pro-ANP for the listed clinical variables (upper panel: age and sex-unadjusted model, lower panel: age and sex-adjusted model). The association between \%pro-ANP of all variables persisted even after the adjustments for age and sex.

\section{DISCUSSION}

On admission, in a vulnerable state of ADHF, there is still an unmet need for a biomarker for prediction of future clinical outcomes. In the present study, we used highly sensitive and specific immunoassays to measure six forms of ANP and BNP in patients with ADHF on their hospital admission and to determine the peptides' associations with clinical data. Among the six peptide forms, pro-ANP and NT-pro-BNP levels on admission were predictive of future adverse clinical events in patients with ADHF (table 1), even compared with total BNP and pro-BNP
Table 4 Univariate/multivariate analysis for \%pro-ANP on admission

\begin{tabular}{lll}
\hline Variables & PE & P value \\
\hline BMI & -0.77 & 0.004 \\
\hline LVDd, mm & 0.21 & 0.033 \\
\hline LVEF, \% & -0.15 & 0.024 \\
\hline BUN, $\mathrm{mg} / \mathrm{dL}$ & 0.13 & 0.044 \\
\hline eGFR, $\mathrm{mL} / \mathrm{min} / 1.73 \mathrm{~m}^{2}$ & -0.13 & 0.008 \\
\hline Total BNP & 0.03 & 0.004 \\
Age and sex-adjusted model & & \\
\hline BMl & -0.77 & 0.006 \\
\hline LVDd, mm & 0.26 & 0.013 \\
\hline LVEF, \% & -0.19 & 0.011 \\
\hline BUN, $\mathrm{mg} / \mathrm{dL}$ & 0.13 & 0.044 \\
\hline eGFR, $\mathrm{mL} / \mathrm{min} / 1.73 \mathrm{~m}^{2}$ & -0.13 & 0.012 \\
\hline Total BNP & 0.03 & 0.004 \\
\hline
\end{tabular}

BMI, body mass index; BNP, B-type natriuretic peptide; BUN, blood urea nitrogen; LVDd, left ventricular end-diastolic diameter; LVEF, left ventricular ejection fraction; PE, parameter estimate; eGFR, estimated glomerular filtration rate.

levels. Comprehensive analyses clearly showed that a higher pro-ANP-to-total ANP ratio (\% pro-ANP) is associated with both larger LV size and lower LVEF, which suggests that higher pro-ANP levels are associated with more advanced LV remodelling (tables 3 and 4).

\section{Differences in the synthesis, secretion and metabolism of ANP and BNP}

Although ANP and BNP activate the same transmembrane receptor (natriuretic peptide receptor-A) and exert similar physiological effects, the regulation of their gene expression, post-translational processing, secretion and metabolism differs. Pro-ANP is stored in secretory granules in atrial myocytes as the prohormone pro-ANP. By contrast, BNP is predominantly secreted from the ventricular myocardium via a constitutive pathway and is present as a mixture of pro-BNP and mature BNP. ${ }^{15} 16$

In patients with $\mathrm{HF}$, the cardiac secretion profile for ANP reportedly differs from that in healthy subjects. By measuring and comparing samples collected from the aortic root, coronary sinus and anterior interventricular vein, Yasue et $\mathrm{l}^{17}$ showed that plasma ANP levels increase in proportion to the severity of LV dysfunction and haemodynamic disorder. The precise mechanisms responsible for ventricular dominancy for ANP synthesis in these patients remain uncertain even at present. Earlier studies indicate that ANP is abundantly expressed in human ventricles during fetal stages, but its expression in the developing heart declines as gestation progresses. ${ }^{18}{ }^{19}$ Interestingly, these reports indicated that ANP production and secretion shift from the atria to the ventricles in patients with severe HF. ${ }^{19}$ Several other groups also reported marked elevation in ventricular ANP expression in patients with dilated cardiomyopathy. ${ }^{20-22}$ It is well accepted that a 
predominance of the fetal gene programme is observed in failing human hearts. ${ }^{1516}$ The evidence that ventricular expression and secretion of ANP is increased in patients with severe HF appears consistent with a return to the fetal gene programme, although a distribution of each molecular form of ANP secreted into the circulation in severe HF remains unknown. Recently, Nishikimi et $a l^{23}$ reported that pro-ANP is the major ANP form secreted from cultured rat neonatal ventricular myocytes $(>50 \%$ of total ANPs), while $\alpha$-ANP was dominant in cultured rat fetal atrial myocytes. Collectively, these results suggest that increased ventricular synthesis and secretion of pro-ANP in patients with severe HF may result in the strong association of plasma pro-ANP levels with LV dysfunction and larger LV size. In the present study, we found that \%proANP (pro-ANP-to-total ANP ratio) is also associated with LV size and function in patients with ADHF (tables 3 and 4). To the best of our knowledge, this is the first study to demonstrate the association of \% pro-ANP with cardiac structure and function. Because these findings suggest dysregulation of pro-ANP secretion or metabolism in patients with ADHF, it will be necessary to investigate the molecular mechanism that makes pro-ANP dominant in the plasma of patients with advanced LV remodelling. On the other hand, atrial cardiomyocytes store high levels of pro-ANP in granules, and previous reports showed that plasma ANP responds more quickly than BNP to acute physiological changes, such as those induced by exercise $^{24}$ or experimental pacing stimulation. ${ }^{25}$ However, given the strong association between pro-ANP levels and ventricular variables detected, rather than left atrial size, in the present study, these findings could indicate that ANP synthesis and secretion from the ventricle and dysregulation of pro-ANP processing, rather than the early responsiveness of ANP secreted from atrium, contribute to the elevation in plasma pro-ANP levels and the higher \% pro-ANP. These findings also raise the possibility that pro-ANP could be a biomarker of a ventricular 'trait' rather than a haemodynamic marker such as BNP levels, but further investigation will be necessary to test that idea.

\section{Comparing prognostic power of ANP and BNP}

Tsutamoto $e t a l^{26}$ reported that plasma BNP is more useful than ANP for assessing mortality among patients with CHF. This is generally explained by the longer halflife of BNP (half-life: 12 to22 min) as compared with ANP (half-life: $2-5 \mathrm{~min}) .{ }^{27} \mathrm{BNP}$ or NT-pro-BNP levels during chronic phases of HF (patients at discharge from hospital or ambulatory patients) are now well established as prognostic biomarkers, but BNP on admission is less predictive in patients with ADHF. Because earlier studies mainly focused on patients during the chronic phase of HF, there has been little data available to compare the prognostic power of ANP and BNP, especially for their various molecular forms, including precursors. The detection of a strong association between plasma pro-ANP levels and long-term clinical outcomes in the present study may shed new light on these issues.

This study showed that pro-ANP has a prognostic power comparable with NT-pro-BNP, and interestingly, multivariate analysis showed that statistical significance of prognostic power of NT-pro-BNP was diminished in the multivariate analysis adjusted by eGFR, but that of pro-ANP persisted in the same analysis. These findings suggest that NT-pro-BNP provides the information, but dependent of renal function. In contrast, pro-ANP is statistically independent of renal function in this study, and these findings suggest that pro-ANP provides the information of ventricular quality independent of renal function compared with NT-pro-BNP. Additionally, the analysis in this study also demonstrated that NT-pro-BNP, but not BNP, was associated with clinical outcomes in patients with ADHF. Several previous studies compared the prognostic power of NT-pro-BNP and BNP in patients with $\mathrm{HF}$ on hospital admission ${ }^{28}$ and before discharge. ${ }^{29}$ One study showed the similar tendency to this study in that NT-pro-BNP had the stronger prognostic power for clinical outcomes in patients with ADHF on hospital admission. ${ }^{28}$

After treatment of ADHF, plasma levels of ANP and BNP levels often greatly decline with haemodynamic improvement. Therefore, natriuretic peptide levels detected after normalisation of haemodynamics or removal of excess body fluid volume may indicate the severity of the HF itself; in other words, only after removal of excess body fluid BNP levels are predictive of future clinical outcomes. BNP levels before discharge from the hospital, thus, reflect the haemodynamic state after removal of excess body fluid, which would explain why levels measured during the acute phase of HF are less predictive. It may be that in the presence of excess fluid volume, BNP levels do not reflect the 'true ventricular trait'. By contrast, our results suggest that pro-ANP levels at admission are robustly associated with ventricular function and size, suggesting a reflection of the qualitative abnormality of the ventricle, which is why pro-ANP at admission may be indicative of prognosis.

\section{Limitations}

The present study has several limitations. First, it was a single-centre investigation performed with a limited number of patients. Second, we excluded patients who had received carperitide before blood collection because we could not exclude the possibility that carperitide affects metabolism of pro-ANP, which could cause selection bias. Third, a system that measures $\alpha$-ANP specifically has not been established, plasma $\alpha$-ANP levels remain uncertain in this study. Further investigation will be necessary to measure all natriuretic peptides including $\alpha$-ANP. Percent pro-ANP indicates a ratio of uncleaved pro-ANP to total ANP. In the clinical settings, it has been hard to measure the pro-ANP processing in patients with acute HF because of a lack of a clinically available assay for $\alpha$-ANP measurements. Therefore, it is impossible to 
elucidate the pro-ANP processing directly at present. Based on this background, we consider \%pro-ANP as an estimated index of unprocessed pro-ANP rate. Since it is controversial to determine that \%pro-ANP (median: $9 \%$ ) is corresponding to pro-ANP processing in the clinical settings, further investigation will be necessary to confirm this issue. Meanwhile, because of the strong relationship of \% pro-ANP with ventricular systolic function, rather, this value can be a possible candidate to indicate the ventricular quality. Fourth, the statistical difference in the prognostic power of the natriuretic peptides was moderate. Importantly, larger cohort studies with patients with $\mathrm{ADHF}$ are necessary to determine the superiority of prognostic power of pro-ANP to that of NT-pro-BNP in patients with acute HF on hospital admission. Lastly, we could not collect the sufficient number of patients with $\mathrm{HF}$ with preserved $\mathrm{EF}$ for the statistical analysis (HFpEF, $\mathrm{n}=29$ ). Because quarter to half of patients with HF are categorised as $\mathrm{HFpEF}^{30}{ }^{31}$ careful interpretation will be necessary to expand these findings to entire patients with HF.

\section{CONCLUSION}

In summary, we were able to measure circulating levels of six molecular forms of ANP and BNP, including pro-ANP, $\beta$-ANP and total ANP, during the acute phase of HF. Our findings demonstrate that pro-ANP levels on admission have a close association with the degree of LV remodelling and are therefore strongly predictive of outcome in patients with ADHF, with independency of renal function. Consequently, pro-ANP may be a better prognostic marker than BNP and NT-pro-BNP during the acute phase of HF.

\section{Author affiliations}

${ }^{1}$ Department of Cardiovascular Medicine, National Cerebral and Cardiovascular Center, Suita, Japan

${ }^{2}$ Department of Cardiovascular Medicine, Kyoto University Graduate School of Medicine, Kyoto, Japan

${ }^{3}$ Department of Molecular Pharmacology, National Cerebral and Cardiovascular Center, Suita, Japan

${ }^{4}$ Omics Research Center, National Cerebral and Cardiovascular Center, Suita, Japan ${ }^{5}$ Research Institute, National Cerebral and Cardiovascular Center, Suita, Japan ${ }^{6}$ Department of Cardiovascular Medicine, Hokkaido University Graduate School of Medicine, Sapporo, Japan

Acknowledgements The authors thank to Tomomi Takahashi and Mitsuko Nakatani of the Department of Molecular Pharmacology for assistance with management of this study and with sample management and measurements of BNPs.

Contributors HT, TA and NM contributed to design of the work; ST, HT, TH, CN-O, $\mathrm{AM}, \mathrm{MA}, \mathrm{YH}, \mathrm{AO}, \mathrm{MA}, \mathrm{TH}$ and $\mathrm{HK}$ contributed to the acquisition and analysis of data for the work; YN, AO, SY, KK and Cl contributed to interpretation of data for the work; ST, HT, TN, YN, SY, NM and Cl contributed to drafting the work or revising it critically for important intellectual content; Final approval of the version was done by HT.

Funding This work was supported in part by the Intramural Research Fund of the National Cerebral and Cardiovascular Center of Japan (grants 22-1-4 and 27-1-5 to N.M.) and a Grant-in-aid for Scientific Research from the Japan Society for the Promotion of Science (grant number: 18K08057 to H.T).

Competing interests None declared.
Patient consent for publication Not required.

Ethics approval This study was approved by our institutional ethics committee (M23-090) and was conducted in accordance with the Declaration of Helsinki.

Provenance and peer review Not commissioned; externally peer reviewed.

Data availability statement All data relevant to the study are included in the article or uploaded as online supplementary information.

Open access This is an open access article distributed in accordance with the Creative Commons Attribution Non Commercial (CC BY-NC 4.0) license, which permits others to distribute, remix, adapt, build upon this work non-commercially, and license their derivative works on different terms, provided the original work is properly cited, appropriate credit is given, any changes made indicated, and the use is non-commercial. See: http://creativecommons.org/licenses/by-nc/4.0/.

\section{REFERENCES}

1. Nishikimi T, Kuwahara K, Nakao K. Current biochemistry, molecular biology, and clinical relevance of natriuretic peptides. J Cardiol 2011;57:131-40.

2. Wei CM, Kao PC, Lin JT, et al. Circulating beta-atrial natriuretic factor in congestive heart failure in humans. Circulation 1993;88:1016-20.

3. Friedl W, Mair J, Thomas S, et al. Natriuretic peptides and cyclic guanosine $3^{\prime}, 5^{\prime}$-monophosphate in asymptomatic and symptomatic left ventricular dysfunction. Heart 1996;76:129-36.

4. Vodovar N, Séronde M-F, Laribi S, et al. Post-translational modifications enhance NT-proBNP and BNP production in acute decompensated heart failure. Eur Heart J 2014;35:3434-41.

5. Takahama H, Takashio S, Nishikimi T, et al. Ratio of pro-B-type natriuretic peptide (BNP) to total BNP is decreased in mild, but not severe, acute decompensated heart failure patients: a novel compensatory mechanism for acute heart failure. Int $\mathrm{J}$ Cardiol 2018;258:165-71.

6. Nakagawa Y, Nishikimi T, Kuwahara K, et al. MiR30-GALNT1/2 Axis-Mediated glycosylation contributes to the increased secretion of inactive human prohormone for brain natriuretic peptide (proBNP) from failing hearts. J Am Heart Assoc 2017;6.

7. Miyata A, Toshimori T, Hashiguchi T, et al. Molecular forms of atrial natriuretic polypeptides circulating in human plasma. Biochem Biophys Res Commun 1987;142:461-7.

8. Nagai-Okatani C, Kangawa K, Takashio S, et al. Novel chemiluminescent enzyme immunoassays for individual quantification of 3 endogenous molecular forms of atrial natriuretic peptide in human plasma. Jrnl App Lab Med 2016;1:47-59.

9. Kociol RD, Horton JR, Fonarow GC, et al. Admission, discharge, or change in B-type natriuretic peptide and long-term outcomes: data from organized program to initiate lifesaving treatment in hospitalized patients with heart failure (OPTIMIZE-HF) linked to Medicare claims. Circ Heart Fail 2011;4:628-36.

10. Khanam SS, Son J-W, Lee J-W, et al. Prognostic value of shortterm follow-up BNP in hospitalized patients with heart failure. BMC Cardiovasc Disord 2017;17.

11. Hamatani $Y$, Nagai T, Shiraishi $Y$, et al. Long-term prognostic significance of plasma B-type natriuretic peptide level in patients with acute heart failure with reduced, mid-range, and preserved ejection fractions. Am J Cardiol 2018;121:731-8.

12. Nishikimi T, Okamoto $H$, Nakamura $M$, et al. Direct immunochemiluminescent assay for proBNP and total BNP in human plasma proBNP and total BNP levels in normal and heart failure. PLoS One 2013;8:e53233.

13. Ho KK, Anderson KM, Kannel WB, et al. Survival after the onset of congestive heart failure in Framingham Heart study subjects. Circulation 1993;88:107-15.

14. Richardson P, McKenna W, Bristow M, et al. Report of the 1995 World health Organization/International Society and Federation of cardiology Task Force on the definition and classification of cardiomyopathies. Circulation 1996;93:841-2.

15. Razeghi P, Young ME, Alcorn JL, et al. Metabolic gene expression in fetal and failing human heart. Circulation 2001;104:2923-31.

16. Rajabi M, Kassiotis C, Razeghi $P$, et al. Return to the fetal gene program protects the stressed heart: a strong hypothesis. Heart Fail Rev 2007;12:331-43.

17. Yasue $H$, Yoshimura $M$, Sumida $H$, et al. Localization and mechanism of secretion of B-type natriuretic peptide in comparison with those of A-type natriuretic peptide in normal subjects and patients with heart failure. Circulation 1994;90:195-203.

18. Bloch KD, Seidman JG, Naftilan JD, et al. Neonatal atria and ventricles secrete atrial natriuretic factor via tissue-specific secretory pathways. Cell 1986;47:695-702. 
19. Tsuchimochi $\mathrm{H}$, Kurimoto F, leki $\mathrm{K}$, et al. Atrial natriuretic peptide distribution in fetal and failed adult human hearts. Circulation 1988;78:920-7.

20. Saito $\mathrm{Y}$, Nakao K, Arai H, et al. Atrial natriuretic polypeptide (ANP) in human ventricle. Increased gene expression of ANP in dilated cardiomyopathy. Biochem Biophys Res Commun 1987;148:211-7.

21. Takemura G, Fujiwara $\mathrm{H}$, Horike $\mathrm{K}$, et al. Ventricular expression of atrial natriuretic polypeptide and its relations with hemodynamics and histology in dilated human hearts. immunohistochemical study of the endomyocardial biopsy specimens. Circulation 1989;80:1137-47.

22. Tarazón E, Roselló-Lletí E, Rivera M, et al. RNA sequencing analysis and atrial natriuretic peptide production in patients with dilated and ischemic cardiomyopathy. PLoS One 2014;9:e90157.

23. Nishikimi T, Nakagawa $\mathrm{Y}$, Minamino N, et al. Pro-B-type natriuretic peptide is cleaved intracellularly: impact of distance between $\mathrm{O}$ glycosylation and cleavage sites. Am J Physiol Regul Integr Comp Physiol 2015;309:R639-R649.

24. Ikeda N, Yasu T, Nishikimi T, et al. N-terminal pro-atrial natriuretic peptide and exercise prescription in patients with myocardial infarction. Regul Pept 2007;141:154-8.

25. Rademaker MT, Charles CJ, Espiner EA, et al. Natriuretic peptide responses to acute and chronic ventricular pacing in sheep. $\mathrm{Am} \mathrm{J}$ Physiol 1996;270:H594-H602.
26. Tsutamoto T, Wada A, Maeda K, et al. Attenuation of compensation of endogenous cardiac natriuretic peptide system in chronic heart failure: prognostic role of plasma brain natriuretic peptide concentration in patients with chronic Symptomatic left ventricular dysfunction. Circulation 1997;96:509-16.

27. Felker GM, Petersen JW, Mark DB. Natriuretic peptides in the diagnosis and management of heart failure. CMAJ 2006;175:611-7.

28. Waldo SW, Beede J, Isakson S, et al. Pro-B-type natriuretic peptide levels in acute decompensated heart failure. J Am Coll Cardiol 2008;51:1874-82.

29. Linssen GCM, Jaarsma T, Hillege HL, et al. A comparison of the prognostic value of BNP versus NT-proBNP after hospitalisation for heart failure. Neth Heart J 2018;26:486-92.

30. Tsuchihashi-Makaya M, Hamaguchi S, Kinugawa S, et al. Characteristics and outcomes of hospitalized patients with heart failure and reduced vs preserved ejection fraction. Report from the Japanese cardiac Registry of heart failure in cardiology (JCARECARD). Circ J 2009;73:1893-900.

31. Owan TE, Hodge DO, Herges RM, et al. Trends in prevalence and outcome of heart failure with preserved ejection fraction. $N$ Engl $J$ Med 2006;355:251-9. 